PROCEEDINGS OF THE

AMERICAN MATHEMATICAL SOCIETY

Volume 125, Number 10, October 1997, Pages 2975-2979

S $0002-9939(97) 04270-6$

\title{
BOHR'S POWER SERIES THEOREM IN SEVERAL VARIABLES
}

\author{
HAROLD P. BOAS AND DMITRY KHAVINSON
}

(Communicated by Theodore W. Gamelin)

\begin{abstract}
Generalizing a classical one-variable theorem of Bohr, we show that if an $n$-variable power series has modulus less than 1 in the unit polydisc, then the sum of the moduli of the terms is less than 1 in the polydisc of radius $1 /(3 \sqrt{n})$.
\end{abstract}

How large can the sum of the moduli of the terms of a convergent power series be? Harald Bohr addressed this question in 1914 with the following remarkable result on power series in one complex variable.

Theorem 1 (Bohr). Suppose that the power series $\sum_{k=0}^{\infty} c_{k} z^{k}$ converges for $z$ in the unit disk, and $\left|\sum_{k=0}^{\infty} c_{k} z^{k}\right|<1$ when $|z|<1$. Then $\sum_{k=0}^{\infty}\left|c_{k} z^{k}\right|<1$ when $|z|<1 / 3$. Moreover, the radius $1 / 3$ is the best possible.

Bohr's paper [2], compiled by G. H. Hardy from correspondence, indicates that Bohr initially obtained the radius $1 / 6$, but this was quickly improved to the sharp result by M. Riesz, I. Schur, and N. Wiener, independently. Bohr's paper presents both his own proof and Wiener's. Some years later, S. Sidon gave a different proof [9], which was subsequently rediscovered by M. Tomić [10].

In this note, we formulate a version of Bohr's theorem in higher dimensions. We write an $n$-variable power series $\sum_{\alpha} c_{\alpha} z^{\alpha}$ using the standard multi-index notation: $\alpha$ denotes an $n$-tuple $\left(\alpha_{1}, \alpha_{2}, \ldots, \alpha_{n}\right)$ of nonnegative integers, $|\alpha|$ denotes the sum $\alpha_{1}+\cdots+\alpha_{n}$ of its components, $\alpha$ ! denotes the product $\alpha_{1} ! \alpha_{2} ! \ldots \alpha_{n}$ ! of the factorials of its components, $z$ denotes an $n$-tuple $\left(z_{1}, \ldots, z_{n}\right)$ of complex numbers, and $z^{\alpha}$ denotes the product $z_{1}^{\alpha_{1}} z_{2}^{\alpha_{2}} \ldots z_{n}^{\alpha_{n}}$.

Let $K_{n}$ denote the $n$-dimensional Bohr radius: the largest number such that if $\sum_{\alpha} c_{\alpha} z^{\alpha}$ converges in the unit polydisc $\left\{\left(z_{1}, \ldots, z_{n}\right): \max _{1 \leq j \leq n}\left|z_{j}\right|<1\right\}$, and if $\left|\sum_{\alpha} c_{\alpha} z^{\alpha}\right|<1$ in the unit polydisc, then $\sum_{\alpha}\left|c_{\alpha} z^{\alpha}\right|<1$ when $\max _{1 \leq j \leq n}\left|z_{j}\right|<K_{n}$.

It is evident from Bohr's one-dimensional result that $K_{n} \leq 1 / 3$ for every $n$. Surprisingly, $K_{n}$ tends to 0 when $n$ increases. Our result implies that the decay rate of $K_{n}$ is essentially $1 / \sqrt{n}$.

Theorem 2. When $n>1$, the $n$-dimensional Bohr radius $K_{n}$ satisfies

$$
\frac{(1 / 3)}{\sqrt{n}}<K_{n}<\frac{2 \sqrt{\log n}}{\sqrt{n}}
$$

Received by the editors May 8, 1996

1991 Mathematics Subject Classification. Primary 32A05.

The first author's research was supported in part by NSF grant number DMS 9500916 and in part at the Mathematical Sciences Research Institute by NSF grant number DMS 9022140.

(C)1997 American Mathematical Society 
Although the theorem gives tight control on the Bohr radius, there is a small amount of slack that we have not been able to remove.

Open question. What is the exact value of the Bohr radius $K_{n}$ when $n>1$ ?

There is an analogous concept of a Bohr radius for the unit ball, or more generally for domains of convergence for power series, that is, for logarithmically convex Reinhardt domains (for definitions, see, for example, [6, section 2.3]). It is easy to see that the polydisc constant $K_{n}$ is a universal lower bound for the Bohr radius of every multi-circular domain (whether logarithmically convex or not).

Theorem 3. Suppose that the $n$-variable power series $\sum_{\alpha} c_{\alpha} z^{\alpha}$ converges for $z$ in a complete Reinhardt domain $G$, and $\left|\sum_{\alpha} c_{\alpha} z^{\alpha}\right|<1$ when $z \in G$. Then $\sum_{\alpha}\left|c_{\alpha} z^{\alpha}\right|<$ 1 when $z$ is in the scaled domain $K_{n} \cdot G$, where $K_{n}$ is the Bohr radius for the unit polydisc.

Indeed, a linear change of variables shows that Theorem 3 holds when $G$ is any bounded polydisc (with its $n$ radii not necessarily equal to each other). By definition, a complete Reinhardt domain $G$ is a union of polydiscs centered at the origin, so $G$ inherits the conclusion of the theorem from the polydisc case.

Proof of Theorem 2. Concerning the lower bound, we will prove more than is stated in the theorem: namely, if $f(z)=\sum_{\alpha} c_{\alpha} z^{\alpha}$ is an analytic function of modulus less than 1 in the unit polydisc, then $\sum_{\alpha}\left|c_{\alpha} z^{\alpha}\right|<1$ in the ball of radius $1 / 3$ centered at the origin. This ball evidently contains the polydisc $\left\{z: \max _{1 \leq j \leq n}\left|z_{j}\right|<\right.$ $1 /(3 \sqrt{n})\}$, whence $K_{n} \geq 1 /(3 \sqrt{n})$. (It will become apparent below why strict inequality obtains.)

We begin by establishing bounds on the coefficients $c_{\alpha}$. By Cauchy's estimate, $\left|c_{\alpha}\right| \leq 1$ for every $\alpha$. We improve this estimate for $\alpha$ different from the 0 multi-index by an argument analogous to one used by Wiener in the single-variable case.

Let $\omega$ denote a primitive $k$ th root of unity, and let $g$ be defined by $g(z)=$ $k^{-1} \sum_{j=1}^{k} f\left(\omega^{j} z\right)$. The modulus of $g$ is again less than 1 in the unit polydisc, and the Taylor series of $g$ begins $c_{0}+\sum_{|\alpha|=k} c_{\alpha} z^{\alpha}+\cdots$ (because the nonconstant terms of homogeneity less than $k$ average out). Next define $h$ by composing $g$ with a linear fractional transformation: $h(z)=\left(g(z)-c_{0}\right) /\left(1-\bar{c}_{0} g(z)\right)$. The modulus of $h$ is less than 1 in the unit polydisc, and the Taylor series of $h$ begins $\sum_{|\alpha|=k} b_{\alpha} z^{\alpha}+\cdots$, where the coefficients of the leading terms (the ones of homogeneity $k$ ) satisfy $b_{\alpha}=c_{\alpha} /\left(1-\left|c_{0}\right|^{2}\right)$. Since the modulus of $h$ does not exceed 1 in the unit polydisc, neither does its $L^{2}$ norm on the unit torus. (We normalize Lebesgue measure on the unit torus to have total mass 1.) The monomials $z^{\alpha}$ are orthonormal on the unit torus, so we have (in particular) that $\sum_{|\alpha|=k}\left|b_{\alpha}\right|^{2} \leq 1$, whence $\left(\sum_{|\alpha|=k}\left|c_{\alpha}\right|^{2}\right)^{1 / 2} \leq$ $\left(1-\left|c_{0}\right|^{2}\right)$.

The Cauchy-Schwarz inequality now implies that

$$
\sum_{\alpha}\left|c_{\alpha} z^{\alpha}\right|=\left|c_{0}\right|+\sum_{k=1}^{\infty} \sum_{|\alpha|=k}\left|c_{\alpha} z^{\alpha}\right| \leq\left|c_{0}\right|+\left(1-\left|c_{0}\right|^{2}\right) \sum_{k=1}^{\infty}\left(\sum_{|\alpha|=k}\left|z^{\alpha}\right|^{2}\right)^{1 / 2} .
$$

But $\sum_{|\alpha|=k}\left|z^{\alpha}\right|^{2} \leq\left(\sum_{j=1}^{n}\left|z_{j}\right|^{2}\right)^{k}$, so if $z$ lies in the ball of radius $1 / 3$, then

$$
\sum_{\alpha}\left|c_{\alpha} z^{\alpha}\right| \leq\left|c_{0}\right|+\left(1-\left|c_{0}\right|^{2}\right) \sum_{k=1}^{\infty} \frac{1}{3^{k}}=\left|c_{0}\right|+\frac{1}{2}\left(1-\left|c_{0}\right|^{2}\right) .
$$


The right-hand side of (2) does not exceed 1 , whatever the value of $c_{0}$. Thus $\sum_{\alpha}\left|c_{\alpha} z^{\alpha}\right|<1$ in the ball of radius $1 / 3$, and so $K_{n} \geq 1 /(3 \sqrt{n})$.

If $k>1$ and at least two of the coordinates $z_{j}$ are nonzero, then $\sum_{|\alpha|=k}\left|z^{\alpha}\right|^{2}$ is strictly less than $\left(\sum_{j=1}^{n}\left|z_{j}\right|^{2}\right)^{k}$. This means that the set of $z$ for which (2) holds is a logarithmically convex Reinhardt domain slightly fatter than the ball of radius $1 / 3$. Hence $K_{n}$ strictly exceeds $1 /(3 \sqrt{n})$ when $n>1$.

We now turn to the right-hand inequality in the theorem. According to the theory of random trigonometric series in $n$ variables (specifically, [5, Theorem 4 of Chapter 6]), there is a constant $C$ such that for every collection of complex numbers $c_{\alpha}$ and every integer $M$ greater than 1, there is a choice of plus and minus signs for which the supremum of the modulus of $\sum_{|\alpha|=M} \pm c_{\alpha} z^{\alpha}$ in the unit polydisc is no more than $C\left(n \sum_{|\alpha|=M}\left|c_{\alpha}\right|^{2} \log M\right)^{1 / 2}$. We emphasize that $C$ is independent of the dimension $n$ and the degree $M$.

If $r$ is any number less than the Bohr radius $K_{n}$, then it follows from this estimate that

$$
r^{M} \sum_{|\alpha|=M}\left|c_{\alpha}\right| \leq C\left(n \sum_{|\alpha|=M}\left|c_{\alpha}\right|^{2} \log M\right)^{1 / 2}
$$

In (3), we take $c_{\alpha}$ equal to $M ! / \alpha$ !, observing that $\sum_{|\alpha|=M}(M ! / \alpha !)=n^{M}$ on the left-hand side. On the right-hand side, we crudely estimate $\sum_{|\alpha|=M}(M ! / \alpha !)^{2} \leq$ $M ! \sum_{|\alpha|=M}(M ! / \alpha !)=M ! n^{M}$. Consequently, we obtain

$$
r^{M} \leq C n^{(1-M) / 2}(M ! \log M)^{1 / 2} .
$$

Taking $M$ th roots in (4) and choosing $M$ to be an integer close to $\log n$, we obtain $K_{n}<C \sqrt{\log n} / \sqrt{n}$ (with a new constant $C$ ).

We now make a rough estimate for $C$. Since $K_{n}$ never exceeds $1 / 3$, the upper bound for $K_{n}$ stated in the theorem is interesting only when $n \geq 189$. If we take $M$ to be the next integer above $\log n$, then $M \geq 6$ for such $n$. Now [5, Theorem 1 of Chapter 6] with $\kappa=3$ and $\rho=\left(2 \pi M^{2}\right)^{n}$ shows the existence of a random homogeneous polynomial $\sum_{|\alpha|=M} \pm(M ! / \alpha !) z^{\alpha}$ whose modulus is bounded on the unit polydisc in dimension $n$ by $3\left(\sum_{|\alpha|=M}(M ! / \alpha !)^{2} n \log \left(6^{1 / n} 2 \pi M^{2}\right)\right)^{1 / 2}$. For the values of $M$ of interest, $6^{1 / n} 2 \pi<M^{2}$, so since $\log M<M$, this upper bound is less than $6\left(M ! n^{M+1} M\right)^{1 / 2}$. Arguing as above, we divide by $n^{M}$ and take the $M$ th root to estimate the Bohr radius. Using that $n^{1 / M}<n^{1 / \log n}=e$, while $M ! M \leq M^{M} 5 ! / 6^{4}$ and $M<(\log n)\left(1+(\log 189)^{-1}\right)$, we find $K_{n}<2 \sqrt{\log n} / \sqrt{n}$ as claimed.

Remark 1. Our method seems unlikely to yield the exact value of the Bohr radius $K_{n}$, but small improvements over Theorem 2 are feasible. For example, the Schwarz lemma for polydiscs (see [8, Lemma 7.5.6]) implies that $\sum_{|\alpha|=1}\left|c_{\alpha}\right| \leq$ $\left(1-\left|c_{0}\right|^{2}\right)$; and if $z$ is in the polydisc of radius $r$, then $\sum_{|\alpha|=k}\left|z^{\alpha}\right|^{2} \leq r^{2 k} \sum_{|\alpha|=k} 1=$ $r^{2 k}\left(\begin{array}{c}n+k-1 \\ k\end{array}\right)$. Hence we find in place of equation (2) that the Bohr radius is no smaller than the solution $r$ of the equation $r+\sum_{k=2}^{\infty} r^{k}\left(\begin{array}{c}n+k-1 \\ k\end{array}\right)^{1 / 2}=1 / 2$.

If we put $r=q / \sqrt{n}$ and optimize the value of $q$, we find (for example) that $K_{n}$ exceeds $(2 / 5) / \sqrt{n}$ when $n>1$, and $K_{n}$ exceeds $(1 / 2) / \sqrt{n}$ for sufficiently large $n$.

Also, it is easy to deduce from inequality (4) the asymptotic upper bound $\lim \sup _{n \rightarrow \infty} K_{n} \sqrt{n / \log n} \leq 1$. 
Remark 2. We thank Professor Henry Helson for bringing to our attention a paper of Bohnenblust and Hille [1] that constructs special $M$-linear forms with unimodular coefficients, the forms being bounded on the unit polydisc in dimension $n$ by $n^{(M+1) / 2}$. One might hope similarly to construct symmetric $M$-linear forms with unimodular coefficients, the forms admitting some weaker upper bound

$C^{M} n^{(M+1) / 2}$ for a constant $C$. The homogeneous polynomial associated to such a symmetric form could then be used in our argument above to eliminate the logarithmic factor in the upper bound for the Bohr radius. However, we can prove that no such symmetric $M$-linear form can exist.

In fact, the homogeneous polynomial associated to a symmetric $M$-linear form with unimodular coefficients can be written $\sum_{|\alpha|=M} c_{\alpha} z^{\alpha}$, where $\left|c_{\alpha}\right|=M ! / \alpha$ !. The supremum of such a polynomial dominates its $L^{2}$ norm on the unit torus, namely $\left(\sum_{|\alpha|=M}(M ! / \alpha !)^{2}\right)^{1 / 2}$, which in turn exceeds $\left(\begin{array}{c}M+n-1 \\ M\end{array}\right)^{-1 / 2} n^{M}$ by the CauchySchwarz inequality applied to $\sum_{|\alpha|=M}[1 \cdot(M ! / \alpha !)]$. When $n$ is fixed, this lower bound grows faster than $n^{M} / M^{n / 2}$ as $M \rightarrow \infty$. Hence the modulus of the polynomial cannot admit an upper bound on the $n$-dimensional unit polydisc of the form $C^{M} n^{(M+1) / 2}$ with $C$ independent of $n$ and $M$.

Remark 3. In [3] (see also [4]), S. Dineen and R. M. Timoney state a result (Theorem 3.2) that, specialized to polydiscs whose $n$ radii are all equal, says $K_{n} \leq$ $2 / \sqrt{n}$, a better asymptotic upper bound than the one in our Theorem 2 . However, the supporting Lemma 3.3 in [3] is false: it claims the existence of a symmetric $M$-linear form with unimodular coefficients that admits an upper bound $2^{M+1}\left[M n^{M+1} \log (1+4 M)\right]^{1 / 2}+1$ on the unit polydisc in dimension $n$; but Remark 2 shows that no such form can exist when the dimension $n$ is large. The error in [3] results from a mistaken assumption that the constants in the estimates of A. M. Mantero and A. Tonge [7] for norms of random tensors carry over unchanged to the case of symmetric tensors. Adjustments to the proof in [7] are required in the symmetric case because the random components of the tensors are no longer independent random variables: see [11] for a clear exposition of random symmetric tensors of order three. After appropriate modifications, the method of Dineen and Timoney does yield that $K_{n}=O\left(n^{\epsilon-1 / 2}\right)$ for an arbitrary positive $\epsilon$, and this is good enough for their applications.

\section{REFERENCES}

[1] H. F. Bohnenblust and Einar Hille, On the absolute convergence of Dirichlet series, Ann. of Math. (2) 32 (1931), 600-622.

[2] Harald Bohr, A theorem concerning power series, Proc. London Math. Soc. (2) 13 (1914), $1-5$.

[3] Seán Dineen and Richard M. Timoney, Absolute bases, tensor products and a theorem of Bohr, Studia Math. 94 (1989), 227-234. MR 91e:46006

[4] _ On a problem of H. Bohr, Bull. Soc. Roy. Sci. Liège 60 (1991), no. 6, 401-404. MR 93e: 46050

[5] Jean-Pierre Kahane, Some random series of functions, second ed., Cambridge University Press, 1985. MR 87 m:60119

[6] Steven G. Krantz, Function theory of several complex variables, second ed., Wadsworth \& Brooks/Cole, Pacific Grove, CA, 1992. MR 93c:32001

[7] Anna Maria Mantero and Andrew Tonge, The Schur multiplication in tensor algebras, Studia Math. 68 (1980), no. 1, 1-24. MR 81k:46074

[8] Walter Rudin, Function theory in polydiscs, Benjamin, New York, 1969. MR 41:501

[9] S. Sidon, Über einen Satz von Herrn Bohr, Math. Z. 26 (1927), 731-732. 
[10] M. Tomić, Sur un théorème de H. Bohr, Math. Scand. 11 (1962), 103-106. MR 31:316

[11] N. Th. Varopoulos, On an inequality of von Neumann and an application of the metric theory of tensor products to operators theory, J. Funct. Anal. 16 (1974), 83-100. MR 50:8116

Department of Mathematics, Texas A\&M University, College Station, Texas 778433368

E-mail address: boas@math.tamu.edu

URL: http://www.math.tamu.edu/ harold.boas/

Department of Mathematical Sciences, University of Arkansas, Fayetteville, ARKANSAS 72701

E-mail address: dmitry@comp.uark.edu

$U R L:$ http://www. uark.edu/depts/mathinfo/people/khavinson/ 